\title{
Observation of thermally activated domain wall transformations
}

\author{
M. Laufenberg, D. Backes, ${ }^{\text {a) }}$ W. Bührer, D. Bedau, M. Kläui, ${ }^{\text {b) }}$ and U. Rüdiger \\ Fachbereich Physik, Universität Konstanz, 78457 Konstanz, Germany \\ C. A. F. Vaz and J. A. C. Bland \\ Cavendish Laboratory, University of Cambridge, Madingley Road, Cambridge, \\ CB3 OHE, United Kingdom \\ L. J. Heyderman and F. Nolting \\ Paul Scherrer Institut, 5232 Villigen PSI, Switzerland
}

S. Cherifi, ${ }^{\text {c) }}$ A. Locatelli, R. Belkhou, ${ }^{\text {d) }}$ and S. Heun ${ }^{\mathrm{e})}$

Sincrotrone Trieste, 34012 Basovizza, Trieste, Italy

E. Bauer

Department of Physics and Astronomy, Arizona State University, Tempe, Arizona 85287-1404

(Received 21 October 2005; accepted 3 January 2006; published online 2 February 2006)

\begin{abstract}
The spin structure of head-to-head domain walls in $\mathrm{Ni}_{80} \mathrm{Fe}_{20}$ structures is studied using high-resolution photoemission electron microscopy. The quantitative phase diagram is extracted from these measurements and found to exhibit two phase boundaries between vortex and transverse domain walls. The results are compared with available theoretical predictions and micromagnetic simulations and differences to the experiment are explained, taking into account thermal excitations. Temperature-dependent measurements show a thermally activated transformation of transverse to vortex domain walls in $7 \mathrm{~nm}$ thick and $730 \mathrm{~nm}$ wide structures at a transition temperature between $260{ }^{\circ} \mathrm{C}$ and $310^{\circ} \mathrm{C}$, which corresponds to a nucleation barrier height for a vortex wall between $6.7 \times 10^{-21} \mathrm{~J}$ and $8.0 \times 10^{-21} \mathrm{~J}$. (C) 2006 American Institute of Physics. [DOI: 10.1063/1.2168677]
\end{abstract}

Domain walls in nanoscale ferromagnetic elements are in the focus of interest because of their potential for applications in a variety of fields like magnetic logic ${ }^{1}$ as well as data storage $^{2}$ and due to their associated fundamental physical effects. $^{3-7}$ Magnetoresistance effects related with domain walls have been investigated in recent years. ${ }^{3,4}$ Domain wall motion induced by external fields ${ }^{5,6}$ has been studied and wall mobilities and depinning fields ${ }^{7}$ have been determined. The interaction between spin polarized current and domain walls (domain wall magnetoresistance and current induced domain wall motion) has been the subject of rising interest recently and was shown to depend critically on the wall spin structure $^{1,3-8}$ with the investigation of critical current densities and of domain wall velocities. The resistivity of a domain wall, its mobility, the depinning fields and critical currents, and the spin torque effect depend critically on the wall spin structure. ${ }^{1,3-8} \mathrm{Ni}_{80} \mathrm{Fe}_{20}$ is a particularly important material in this context ${ }^{1,4,5,7-9}$ and a comprehensive study of the domain wall spin structure in this material therefore is of paramount interest. In this field, temperature and heating effects play a key role, ${ }^{10,11}$ in particular, the wall spin structure was predicted to depend on the temperature. ${ }^{11}$

Ring elements have proven to be a useful geometry for an investigation of domain walls since they can easily be created and positioned by applying an external uniform magnetic field. Ferromagnetic rings can be in the flux closure

\footnotetext{
a) Also at: Paul Scherrer Institut, CH-5232 Villigen PSI.

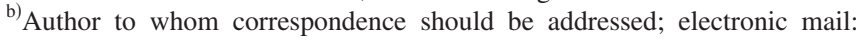
mathias@klaeui.de

${ }^{c}$ Permanent address: LLN-CNRS, BP 166, 38042 Grenoble, France.

d) Permanent address: Synchrotron SOLEIL, 91192 Gif-sur-Yvette, France.

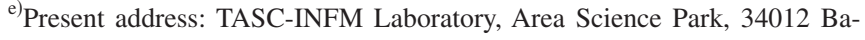
sovizza, Trieste, Italy.
}

vortex state or in the onion state characterized by $180^{\circ}$-headto-head and tail-to-tail domain walls, which can be of either vortex or transverse type ${ }^{12,13}$ with the spin structures shown in Figs. 1(a) and 1(b), respectively. In Co elements, the domain wall type has been determined for various geometries at room temperature and the corresponding phase diagram with a boundary between vortex and transverse walls was extracted. ${ }^{12}$ The results were compared with calculations ${ }^{13}$ as well as micromagnetic simulations and both differed from the experimentally found phase boundary. This was attributed to the fact that vortex and transverse walls constitute local energy minima separated by a barrier that can be overcome by thermal activation, but no direct experimental evidence has been brought forward so far.

In this letter, we systematically study the spin structure of head-to-head domain walls in NiFe at variable temperature by direct imaging. We present the phase diagram and compare the results with available theoretical calculations and the results of micromagnetic simulations. We explore the difference between the experimentally found phase boundary and the $0 \mathrm{~K}$ theory by taking into account the energy land-

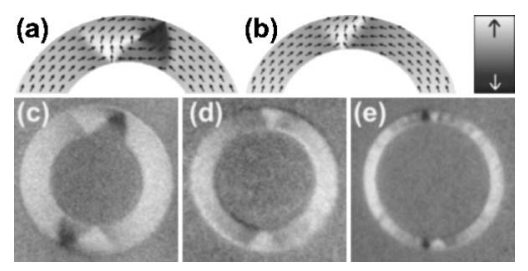

FIG. 1. Spin structure of (a) a vortex wall and (b) a transverse wall simulated using the OOMMF code. PEEM images of (c) a $30 \mathrm{~nm}$ thick and $530 \mathrm{~nm}$ wide $(D=2.7 \mu \mathrm{m}),(\mathrm{d})$ a $10 \mathrm{~nm}$ thick and $260 \mathrm{~nm}$ wide $(D=1.64 \mu \mathrm{m})$, and (e) a $3 \mathrm{~nm}$ thick and $730 \mathrm{~nm}$ wide $(D=10 \mu \mathrm{m})$ ring. The gray scale shows the magnetization direction. 


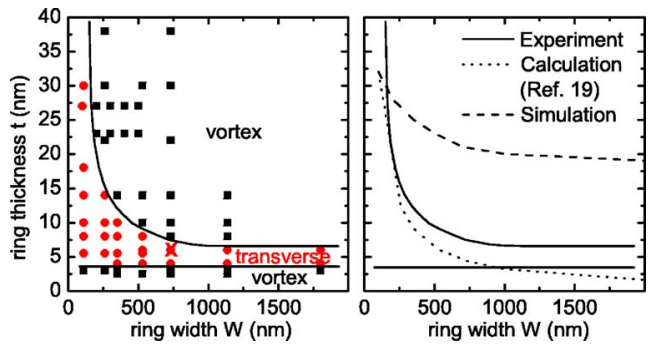

FIG. 2. (Color online) (a) Experimental phase diagram for head-to-head domain walls in $\mathrm{NiFe}$ rings at room temperature. Black squares indicate vortex walls and red circles transverse walls. The phase boundaries are shown as solid lines. (b) A comparison of the upper experimental phase boundary (solid line) with results from calculations (dotted line) and micromagnetic simulations (dashed line). Close to the phase boundaries, both wall types can be observed in nominally identical samples due to slight geometrical variations. The thermally activated wall transitions shown were observed for the ring geometry marked with a red cross $(W=730 \mathrm{~nm}, t=7 \mathrm{~nm})$.

scape involved and relate it to thermally activated transitions from one wall type to another. This allows us to derive a qualitative temperature dependence of the phase boundary and extract the energy barrier height for the vortex nucleation. Furthermore, the low thickness regime $(t<5 \mathrm{~nm})$ of the phase diagram is investigated.

Arrays of polycrystalline NiFe rings with thickness $t$ between 2.5 and $38 \mathrm{~nm}$, width $W$ between 110 and $1800 \mathrm{~nm}$, and outer diameter $D$ between 1.64 and $10 \mu \mathrm{m}$ were fabricated using electron-beam lithography and molecular beam epitaxy with subsequent liftoff, as described in Ref. 14. The edge-to-edge spacing between adjacent rings was more than twice the diameter to prevent dipolar interactions that would otherwise influence the domain wall type. ${ }^{15}$ To determine the spin structure of the domain walls as a function of the ring geometry, the samples were imaged using x-ray magnetic circular dichroism photoemission electron microscopy (XMCD-PEEM) ${ }^{16}$ The secondary electron yield in this microscopy technique is proportional to the dot product of the magnetization $\mathbf{M}$ and the helicity $\mathbf{P}$ of the elliptically polarized monochromatic light from a synchrotron source. The yield difference between opposite helicities is visible as magnetic contrast in the obtained images.

In Fig. 1, we present PEEM images of (c) a thick and wide ring $(t=30 \mathrm{~nm}, W=530 \mathrm{~nm}, D=2.7 \mu \mathrm{m})$, (d) a thin and narrow ring $(t=10 \mathrm{~nm}, W=260 \mathrm{~nm}, D=1.64 \mu \mathrm{m})$ and (e) an ultrathin ring $(t=3 \mathrm{~nm}, W=730 \mathrm{~nm}, D=10 \mu \mathrm{m})$ measured at room temperature. The domain wall type was systematically determined from PEEM images for more than 50 combinations of ring thickness and width and the quantitative phase diagram shown in Fig. 2(a) was extracted. The phase diagram exhibits two phase boundaries indicated by solid lines between vortex walls (thick and wide rings, squares) and transverse walls (thin and narrow rings, circles) and again vortex walls for ultrathin rings. We first discuss the upper boundary that was similarly observed for Co. ${ }^{12}$ To understand theoretically this phase boundary, McMichael and Donahue $^{13}$ calculated the energies for a vortex and a transverse wall and determined the phase boundary by equating these two energies. The calculated boundary is of the form $t \cdot W=C \cdot \delta^{2}$, where $\delta$ is the exchange length and $C$ a universal constant. The phase boundary is shifted to lower thickness and smaller width compared to the experimental boundary as shown by the dotted line in Fig. 2(b). We have suggested ${ }^{12}$ that this discrepancy can be understood by taking into ac-

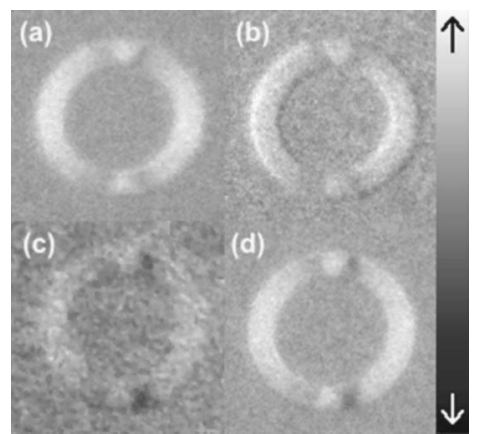

FIG. 3. PEEM images of a $7 \mathrm{~nm}$ thick and $730 \mathrm{~nm}$ wide ring imaged during a heating cycle at temperatures of (a), (d) $T=20^{\circ} \mathrm{C}$ (before and after heating, respectively), (b) $T=260{ }^{\circ} \mathrm{C}$, and (c) $T=310^{\circ} \mathrm{C}$ (estimated errors are $\pm 10 \mathrm{~K}$ ). The two transverse walls (a) are not visibly influenced by heating (b) up to the transition temperature (c), at which a thermally activated transition to a vortex type occurs in both walls. (d) The vortex walls are retained after cooling down. The gray scale shows the magnetization direction.

count the following: The calculations ${ }^{13}$ compare total energies and therefore determine the wall type with the absolute minimum energy as being favorable. In the experiment, the wall type was investigated after saturation of the ring in a magnetic field and relaxing this field to zero. During relaxation, first a transverse wall is formed reversibly. ${ }^{17}$ For the formation of a vortex wall, an energy barrier has to be overcome to nucleate the vortex core. So the observed spin structure does not necessarily constitute the absolute minimum energy, but transverse walls can be observed for combinations of thickness and width, where they constitute local energy minima, even if a vortex wall has a lower energy for this geometry. To shed further light onto this, we have simulated the experiment by calculating the domain wall spin structure after reducing an externally applied field stepwise using the OOMMF $\operatorname{code}^{18} \quad\left(M_{s}=800 \times 10^{3} \mathrm{~A} / \mathrm{m}, A=13\right.$ $\times 10^{-12} \mathrm{~J} / \mathrm{m}, 5 \mathrm{~nm}$ cell size). The simulated boundary is shifted to higher thickness and larger width compared to the experiment. This we attribute to the fact that thermal excitations help to overcome the energy barrier between transverse and vortex walls at room temperature, while they are not taken into account in the $0 \mathrm{~K}$ simulation. So we can expect that for temperatures above room temperature the upper phase boundary is shifted to lower thickness, in other words, that transverse walls formed at room temperature change to vortex walls with rising temperature. This means that with rising temperature the experimental phase boundary approaches the theoretical one since the walls attain the energetically lower spin structure. In order to check this aspect, we have performed temperature-dependent XMCD-PEEM studies. Figure 3 shows an image series of a $7 \mathrm{~nm}$ thick and $730 \mathrm{~nm}$ wide ring for different temperatures of (a), (d) $T$ $=20{ }^{\circ} \mathrm{C}$ (before and after heating), (b) $T=260{ }^{\circ} \mathrm{C}$, and (c) $T=310^{\circ} \mathrm{C}$. Transverse walls are formed (a) during saturation in a magnetic field and relaxation before imaging. At first, heating does not influence the spin structure of the domain walls, as shown in (b), only the image contrast becomes weaker because imaging is more difficult at higher temperatures due to drift problems and decreasing magnetization. At a transition temperature between $T=260{ }^{\circ} \mathrm{C}$ and $T=310^{\circ} \mathrm{C}$ corresponding to a thermal energy between $6.7 \times 10^{-21} \mathrm{~J}$ and $8.0 \times 10^{-21} \mathrm{~J}$, the transverse walls change to vortex walls (c), which means that a domain wall spin structure was created that is not accessible for the same ring geometry by only 
applying uniform magnetic fields. The investigation of a larger number of domain walls shows that the transition temperature slightly varies between different domain walls in rings of the same size. This can be expected because the change of the domain wall spin structure with temperature from transverse to vortex-related with overcoming the energy barrier between the two wall configurations-is a stochastic thermally activated switching process with a distribution of energy barriers, which leads to a variation in the transition temperature. Imperfections of the microstructure may also cause a variation of the transition temperature for nominally identical rings since defects can assist as well as impede the change of the spin structure from transverse to vortex. Figure 3(d) confirms that the vortex wall is stable during cooling down and that a XMCD signal as strong as in (a) is retrieved. This means that both domain wall types are (meta-)stable spin configurations and therefore constitute local energy minima at room temperature. These PEEM experiments directly show that the position of the upper experimental phase boundary is temperature dependent and is shifted to lower thickness and width with increasing temperature. These results thus confirm the hypothesis about the discrepancy between experiment and theory put forward in Ref. 12: Both domain wall types constitute local energy minima, with the transverse wall attained due to the magnetization process, even if a vortex wall has a lower energy. It can be seen by comparing the boundaries from Fig. 2 with the corresponding results in Ref. 12, that for $\mathrm{NiFe}$ the calculations ${ }^{13}$ fit the experiment better than the simulations while for Co it is vice versa. The energy barrier between a transverse and a vortex wall can be overcome more easily in the case of $\mathrm{NiFe}$ rather than $\mathrm{Co}$, so that transverse walls created are more likely to be retained at a certain temperature in a Co ring than in a $\mathrm{NiFe}$ ring with analogous dimensions. This is consistent with the observation that in NiFe there is a more abrupt change between transverse and vortex walls with varying geometry than in Co. ${ }^{15}$ The reason for this difference is thought to be the smaller number of pinning sites in the soft $\mathrm{NiFe}$ fcc crystallites with weak anisotropy compared to the strongly anisotropic hcp Co crystallites that lead to more pinning sites.

We turn now to the discussion of the low thickness regime, where a second phase boundary between 3 and $4 \mathrm{~nm}$ is found. In terms of energetics, this is not expected because the calculations ${ }^{13}$ show that a transverse wall has a lower energy than a vortex wall in this thickness regime. But these calculations assume a perfect microstructure and do not take into account morphological defects such as the surface roughness. Holes that might serve as nucleation centers for the vortex wall formation, were not observed in atomic force microscopy images. However, this does not exclude a spatial modulation of magnetic properties ${ }^{19}$ such as the exchange or the saturation magnetization, which could locally allow a stronger twisting of adjacent spins at a reduced cost of energy. Thus a vortex wall is energetically more favorable in this thickness regime only due to imperfections of the microstructure or the morphology. Since the ultrathin limit was not investigated in Ref. 12 we have performed PEEM imaging also for Co elements in this regime. Images are not shown here, but the measurements clearly indicate the existence of a phase boundary at a similar thickness like in NiFe. This can be expected because the suggested explanation applies for $\mathrm{Co}$ as well as for NiFe.
In conclusion, we have determined the spin structure of domain walls in NiFe by XMCD-PEEM and extracted the corresponding room temperature phase diagram which exhibits two phase boundaries between the wall types. In temperature-dependent XMCD-PEEM imaging, we have observed a thermally activated switching from transverse walls to vortex walls at elevated temperatures at a transition temperature between $260^{\circ} \mathrm{C}$ and $310^{\circ} \mathrm{C}$. This gives direct experimental evidence for the fact that transverse and vortex walls are separated by an energy barrier which can be overcome thermally. The discrepancy between experiment and simulation can now be attributed to thermal excitations, confirming previous conjectures. ${ }^{12}$ The low thickness regime of the phase diagram revealed a second phase boundary, which we attribute to spatial modulations of the magnetic properties in our thinnest samples.

The authors acknowledge support by the Deutsche Forschungsgemeinschaft through SFB 513, by the EPSRC (UK), by the EC through the 6th Framework Program, and by the EU through the European Regional Development Fund (Interreg III A Program). Part of this work was carried out at the Swiss Light Source, Villigen / Switzerland [Fig. 1(e), Fig. 3, data for Fig. 2] and at Elettra, Trieste / Italy [Figs. 1(c) and 1(d), data for Fig. 2].

${ }^{1}$ D. A. Allwood, G. Xiong, C. C. Faulkner, D. Atkinson, D. Petit, and R. P. Cowburn, Science 309, 1688 (2005).

${ }^{2}$ G. A. Prinz, J. Magn. Magn. Mater. 200, 57 (1999).

${ }^{3}$ P. M. Levy and S. Zhang, Phys. Rev. Lett. 79, 5110 (1997); U. Ebels, A. Radulescu, Y. Henry, L. Piraux, and K. Ounadjela, ibid. 84, 983 (2000).

${ }^{4}$ M. Kläui, C. A. F. Vaz, J. Rothman, J. A. C. Bland, W. Wernsdorfer, G. Faini, and E. Cambril, Phys. Rev. Lett. 90, 097202 (2003).

${ }^{5}$ D. Atkinson, D. A. Allwood, G. Xiong, M. D. Cooke, C. C. Faulkner, and R. P. Cowburn, Nat. Mater. 2, 85 (2003).

${ }^{6}$ Y. Nakatani, A. Thiaville, and J. Miltat, Nat. Mater. 2, 521 (2003); R. Wieser, U. Nowak, and K. D. Usadel, Phys. Rev. B 69, 064401 (2004).

${ }^{7}$ M. Kläui, H. Ehrke, U. Rüdiger, T. Kasama, R. E. Dunin-Borkowski, D. Backes, L. J. Heyderman, C. A. F. Vaz, J. A. C. Bland, G. Faini, E. Cambril, and W. Wernsdorfer, Appl. Phys. Lett. 87, 102509, (2005).

${ }^{8}$ A. Yamaguchi, T. Ono, S. Nasu, K. Miyake, K. Mibu, and T. Shinjo, Phys. Rev. Lett. 92, 077205 (2004); M. Kläui, C. A. F. Vaz, J. A. C. Bland, W. Wernsdorfer, G. Faini, E. Cambril, L. J. Heyderman, F. Nolting, and U. Rüdiger, ibid. 94, 106601 (2005).

${ }^{9}$ S. I. Kiselev, J. C. Sankey, I. N. Krivorotov, N. C. Emley, M. Rinkoski, C. Perez, R. A. Buhrman, and D. C. Ralph, Phys. Rev. Lett. 93, 036601 (2004); Y. Huai, F. Albert, P. Nguyen, M. Pakala, and T. Valet, Appl. Phys. Lett. 84, 3118 (2004).

${ }^{10}$ D. Atkinson and R. P. Cowburn, Appl. Phys. Lett. 85, 1386 (2004); A. Yamaguchi, S. Nasu, H. Tanigawa, T. Ono, K. Miyake, K. Mibu, and T. Shinjo, ibid. 86, 012511 (2005).

${ }^{11}$ N. Kazantseva, R. Wieser, and U. Nowak, Phys. Rev. Lett. 94, 037206 (2005).

${ }^{12}$ M. Kläui, C. A. F. Vaz, J. A. C. Bland, L. J. Heyderman, F. Nolting, A. Pavlovska, E. Bauer, S. Cherifi, S. Heun, and A. Locatelli, Appl. Phys. Lett. 85, 5637 (2004).

${ }^{13}$ R. D. McMichael and M. J. Donahue, IEEE Trans. Magn. 33, 4167 (1997).

${ }^{14}$ L. J. Heyderman, C. David, M. Kläui, C. A. F. Vaz, and J. A. C. Bland, J. Appl. Phys. 93, 10011 (2003).

${ }^{15}$ M. Laufenberg, submitted.

${ }^{16}$ J. Stöhr, Y. Wu, B. D. Hermsmeier, M. G. Samant, G. R. Harp, S. Koranda, D. Dunham, and B. P. Tonner, Science 259, 658 (1993).

${ }^{17}$ M. Kläui, C. A. F. Vaz, W. Wernsdorfer, E. Bauer, S. Cherifi, S. Heun, A. Locatelli, G. Faini, E. Cambril, L. J. Heyderman, and J. A. C. Bland, Physica B 343, 343 (2004).

${ }^{18}$ OOMMF is available at http://math.nist.gov/oommf.

${ }^{19}$ I. Hashim, H. S. Joo, and H. A. Atwater, Surf. Rev. Lett. 2, 427 (1994). 